\title{
A New Coupling Reaction of Secondary Amines, Aldehydes and Alkynes Catalyzed by an Iridium Complex
}

Satoshi Sakaguchi*, Tomoya Mizuta, and Yasutaka Ishii*

Department of Applied Chemistry, Faculty of Engineering, Kansai University,

Suita, Osaka 564-8680, Japan

satoshi@ipcku.kansai-u.ac.jp; ishii@ipcku.kansai-u.ac.jp

Supporting Information

Table of contents

S2-3 Experimental Procedures and spectral data

S4 Copies of ${ }^{1} \mathrm{H}$ and ${ }^{13} \mathrm{C}$ NMR spectra for $4 \mathbf{a}(+5 \mathbf{a})$

S5 Copies of ${ }^{1} \mathrm{H}$ and ${ }^{13} \mathrm{C}$ NMR spectra for $4 \mathbf{b}(+5 \mathbf{b})$

S6 Copies of ${ }^{1} \mathrm{H}$ and ${ }^{13} \mathrm{C}$ NMR spectra for $4 \mathbf{c}(+5 \mathrm{c})$

S7 Copy of ${ }^{1} \mathrm{H}$ spectra for $\mathbf{4 a - b}(\mathbf{+ 5 a}-\mathbf{b})$

S8 Copies of ${ }^{1} \mathrm{H}$ and ${ }^{13} \mathrm{C}$ NMR spectra for $4 \mathbf{d d}(+5 \mathbf{d})$

S9 Copy of ${ }^{1} \mathrm{H}$ spectra for $4 \mathbf{e}(+5 \mathbf{e})$

S10 Copies of ${ }^{1} \mathrm{H}$ and ${ }^{13} \mathrm{C}$ NMR spectra for $\mathbf{4 f}(\mathbf{+ 5 f})$

S11 Copies of ${ }^{1} \mathrm{H}$ and ${ }^{13} \mathrm{C}$ NMR spectra for $\mathbf{4 g}(+5 \mathrm{~g})$

S12 Copies of ${ }^{1} \mathrm{H}$ and ${ }^{13} \mathrm{C}$ NMR spectra for $4 \mathbf{h}(+5 \mathbf{h})$ 


\section{Experimental Section}

All starting materials were commercially available and used without any purification. GLC analysis was performed with a flame ionization detector using a $0.2 \mathrm{~mm}$ x $25 \mathrm{~m}$ capillary column (OV-1). ${ }^{1} \mathrm{H}$ - and ${ }^{13} \mathrm{C}-\mathrm{NMR}$ were measured at 270 or $400 \mathrm{MHz}$ and $67.5 \mathrm{MHz}$, respectively, in $\mathrm{CDCl}_{3}$ with $\mathrm{Me}_{4} \mathrm{Si}$ as the internal standard.

General Procedure: To a 1,4-dioxane solution $\left(\begin{array}{llll}2.0 & \mathrm{~mL}) & \text { of }\end{array}\right.$ dichlorobis $(1,5$-cyclooctadiene $)$ diirdium $[\operatorname{IrCl}(\operatorname{cod})]_{2}(0.025 \mathrm{mmol})$ were added $\mathbf{1 a}(0.5 \mathrm{mmol})$, 2a $(0.25 \mathrm{mmol})$ and $3 \mathbf{a}(0.5 \mathrm{mmol})$. Then, the reaction mixture was stirred at $50{ }^{\circ} \mathrm{C}$ for $3 \mathrm{~h}$. The reaction was quenched with wet ether, and the GC and GC-MS analyses were performed. The yields of products were estimated from the peak areas based on the internal standard technique using GC. The product was isolated by column chromatography (230-400 mesh silica gel, $n$-hexane : ethyl acetate $=10-20: 1)$, and the isolated yields of the products were slightly lower than the GC yields.

The product 4 was not isolated as a pure form. The NMR spectra of the product consisted of a stereoisomeric mixture of $\mathbf{4}$, and a small amount of $\mathbf{5}$ has been measured (typically, see the NMR spectra of the product $\mathbf{4 a - b}$, Table 2, Run 4). Therefore, signals on ${ }^{13} \mathrm{C}-\mathrm{NMR}$ could not be assigned completely.

Spectral data for compound 4a: ${ }^{1} \mathrm{H}$ NMR $\delta=5.18(\mathrm{t}, J=7.3 \mathrm{~Hz}, 1 \mathrm{H}), 2.82(\mathrm{~s}, 2 \mathrm{H}), 2.21(\mathrm{t}, J=$ $7.3 \mathrm{~Hz}, 4 \mathrm{H}), 2.00-1.91(\mathrm{~m}, 4 \mathrm{H}), 1.37-1.20(\mathrm{~m}, 18 \mathrm{H}), 0.82(\mathrm{t}, J=7.3 \mathrm{~Hz}, 12 \mathrm{H}) ;{ }^{13} \mathrm{C} \mathrm{NMR} \delta=138.0$, 127.4, 53.5, 52.8, 35.7, 32.0, 30.6-29.5, 29.7, 28.3, 23.4, 22.8, 20.8, 14.2, 13.9; HRMS (EI, M $\left.{ }^{+}\right)$ calcd for $\mathrm{C}_{20} \mathrm{H}_{41} \mathrm{~N}_{1}$ : 295.3239, found: 295.3246 .

Spectral data for compound 4b: ${ }^{1} \mathrm{H}$ NMR $\delta=5.26(\mathrm{t}, J=7.3 \mathrm{~Hz}, 1 \mathrm{H}), 2.90(\mathrm{~s}, 2 \mathrm{H}), 2.27(\mathrm{t}, J=$ $7.3 \mathrm{~Hz}, 4 \mathrm{H}), 2.10-2.02(\mathrm{~m}, 4 \mathrm{H}), 1.47-1.27(\mathrm{~m}, 12 \mathrm{H}), 0.93(\mathrm{t}, J=7.3 \mathrm{~Hz}, 3 \mathrm{H}), 0.89-0.83(\mathrm{~m}, 9 \mathrm{H})$; ${ }^{13} \mathrm{C}$ NMR $\delta=137.4,129.2,55.7,52.6,35.5,31.8,29.2,28.2,22.7,20.8,20.2,14.7,14.1,12.0$. Spectral data for compound 4c: ${ }^{1} \mathrm{H}$ NMR $\delta=5.25(\mathrm{t}, J=7.3 \mathrm{~Hz}, 1 \mathrm{H}), 2.88(\mathrm{~s}, 2 \mathrm{H}), 2.30(\mathrm{t}, J=$ 
$7.3 \mathrm{~Hz}, 4 \mathrm{H}), 2.05-2.02(\mathrm{~m}, 4 \mathrm{H}), 1.40-1.27(\mathrm{~m}, 30 \mathrm{H}), 0.88(\mathrm{t}, J=7.3 \mathrm{~Hz}, 12 \mathrm{H}) ;{ }^{13} \mathrm{C}$ NMR $\delta=137.9$, $127.7,53.8,52.8,35.7,31.9,31.7,29.9,29.2,28.3,27.3,27.1,27.0,22.8,22.6,14.1$.

Spectral data for compound 4a-b (The product in Table 2, Run 4): ${ }^{1} \mathrm{H}$ NMR $\delta=7.31-7.16(\mathrm{~m}$, $5 \mathrm{H}), 5.34(\mathrm{t}, J=7.3 \mathrm{~Hz}, 1 \mathrm{H}), 3.38(\mathrm{~s}, 2 \mathrm{H}), 2.80(\mathrm{~s}, 2 \mathrm{H}), 2.35-2.02(\mathrm{~m}, 6 \mathrm{H}), 1.47-1.27(\mathrm{~m}, 10 \mathrm{H})$, 0.96-0.84 (m, 9H).

Spectral data for compound 5a-b: ${ }^{1} \mathrm{H}$ NMR $\delta=7.31-7.16(\mathrm{~m}, 5 \mathrm{H}), 6.43(\mathrm{~s}, 1 \mathrm{H}), 3.11(\mathrm{~s}, 2 \mathrm{H})$, 2.35-2.02 (m, 6H), 1.47-1.27 (m, 12H), 0.96-0.84 (m, 9H).

Spectral data for compound 4d: ${ }^{1} \mathrm{H}$ NMR $\delta=5.17(\mathrm{t}, J=7.3 \mathrm{~Hz}, 1 \mathrm{H}), 2.68(\mathrm{~s}, 2 \mathrm{H}), 2.18(\mathrm{br}, 4 \mathrm{H})$, 2.18-1.88 (m, 4H), 1.45-1.43 (m, 4H), 1.35-1.21 (m, 12H), $0.82(\mathrm{t}, J=7.3 \mathrm{~Hz}, 3 \mathrm{H}), 0.81(\mathrm{t}, J=$ $7.3 \mathrm{~Hz}, 3 \mathrm{H}) ;{ }^{13} \mathrm{C}$ NMR $\delta=136.9,127.5,66.0,54.6,31.9-22.5,14.2,13.9$.

Spectral data for compound 4e: ${ }^{1} \mathrm{H}$ NMR $\delta=5.27(\mathrm{t}, J=7.3 \mathrm{~Hz}, 1 \mathrm{H}), 2.86(\mathrm{~s}, 2 \mathrm{H}), 2.07-1.25(\mathrm{~m}$, $23 \mathrm{H}), 0.91-0.86(\mathrm{~m}, 9 \mathrm{H})$.

Spectral data for compound 4f: ${ }^{1} \mathrm{H}$ NMR $\delta=5.24(\mathrm{t}, J=7.3 \mathrm{~Hz}, 1 \mathrm{H}), 2.75(\mathrm{~s}, 2 \mathrm{H}), 2.07-1.25(\mathrm{~m}$, 23H), 0.91-0.81 (m, 9H); ${ }^{13} \mathrm{C}$ NMR $\delta=141.3,127.9,65.7,62.1,54.1,33.2-19.7,14.1,13.9$. Spectral data for compound $4 \mathrm{~g}: \quad{ }^{1} \mathrm{H}$ NMR $\delta=5.20(\mathrm{t}, J=7.3 \mathrm{~Hz}, 1 \mathrm{H}), 3.66-3.59(\mathrm{~m}, 4 \mathrm{H}), 2.73(\mathrm{~s}$, $2 \mathrm{H}), 2.28-2.27(\mathrm{~m}, 4 \mathrm{H}), 1.99-1.89(\mathrm{~m}, 4 \mathrm{H}), 1.33-1.21(\mathrm{~m}, 10 \mathrm{H}), 0.82(\mathrm{t}, J=7.3 \mathrm{~Hz}, 3 \mathrm{H}), 0.81(\mathrm{t}$, $J=7.3 \mathrm{~Hz}, 3 \mathrm{H}) ;{ }^{13} \mathrm{C}$ NMR $\delta=136.0,128.6,67.1,65.6,53.6,31.7-22.4,14.0,13.8$.

Spectral data for compound $4 \mathrm{~h}: \quad{ }^{1} \mathrm{H}$ NMR $\delta=7.09-7.00(\mathrm{~m}, 4 \mathrm{H}), 5.34(\mathrm{t}, J=7.3 \mathrm{~Hz}, 1 \mathrm{H}), 3.53(\mathrm{~s}$, 2H), $2.98(\mathrm{~s}, 2 \mathrm{H}), 2.85(\mathrm{t}, J=5.7 \mathrm{~Hz}, 2 \mathrm{H}), 2.62(\mathrm{t}, J=5.7 \mathrm{~Hz}, 2 \mathrm{H}), 2.11-2.03(\mathrm{~m}, 4 \mathrm{H}), 1.41-1.26$ (m, 10H), $0.93(\mathrm{t}, J=7.3 \mathrm{~Hz}, 3 \mathrm{H}), 0.88(\mathrm{t}, J=7.3 \mathrm{~Hz}, 3 \mathrm{H}) ;{ }^{13} \mathrm{C}$ NMR $\delta=136.6-125.3,64.9,56.4$, $50.3,31.9-22.5,14.2,14.0$. 
$4 a$ (with small amount of 5a)
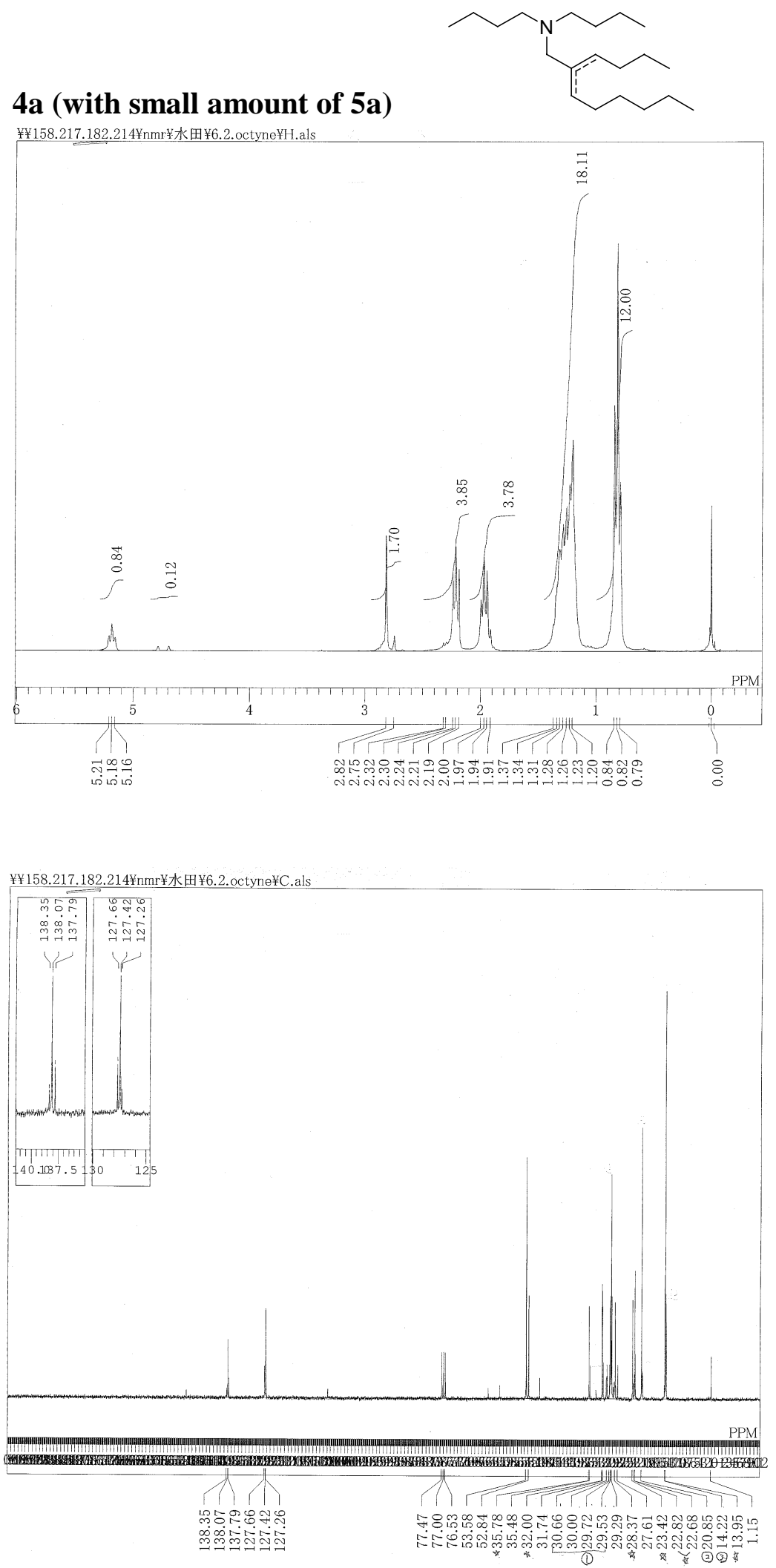


\section{4b (with small amount of 5b)}
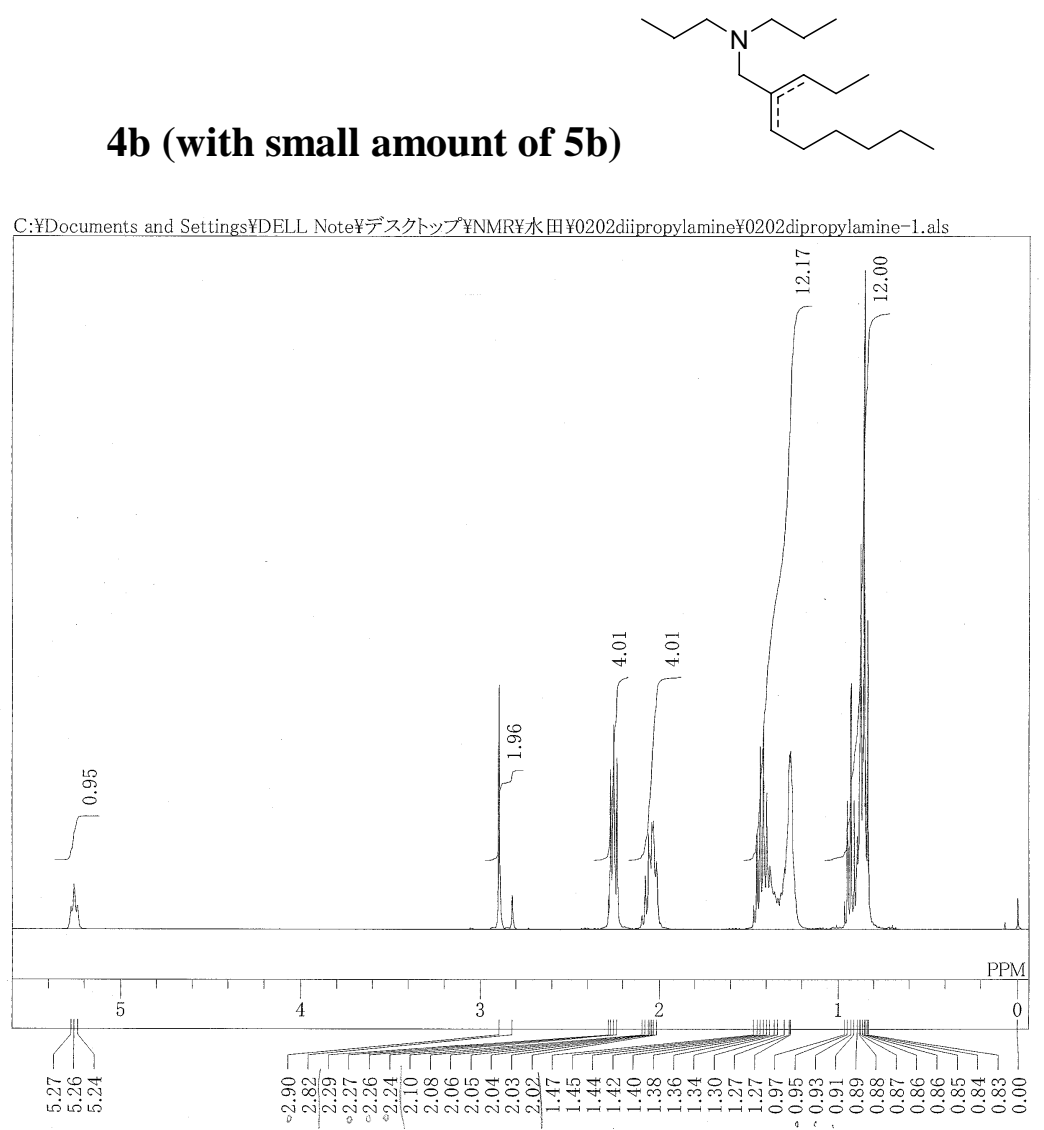

C: ¥Documents and Settings¥DELL Note¥デスクトップ¥NMR¥水田¥0202diipropylamine¥0204dipropylamineC-1.als

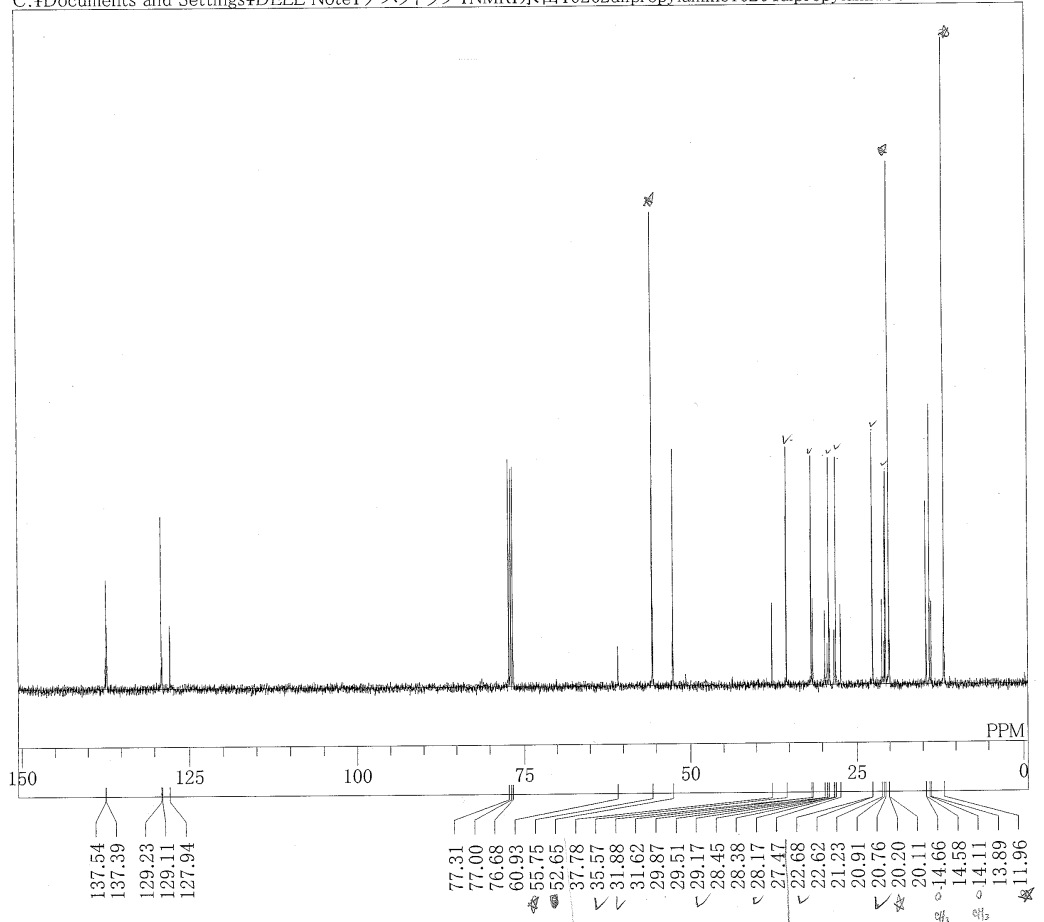



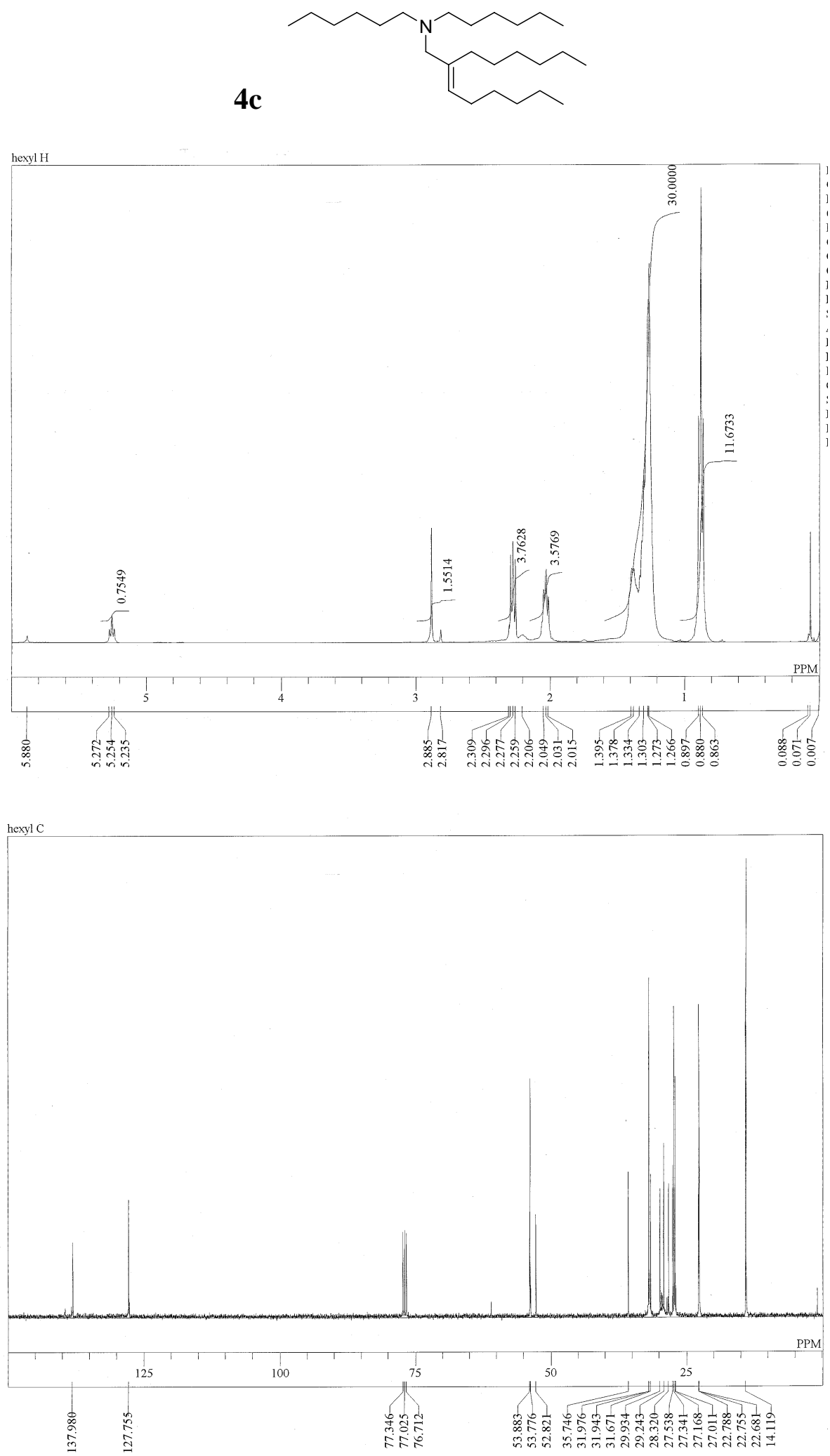
4a-b (with small amount of 5a-b)
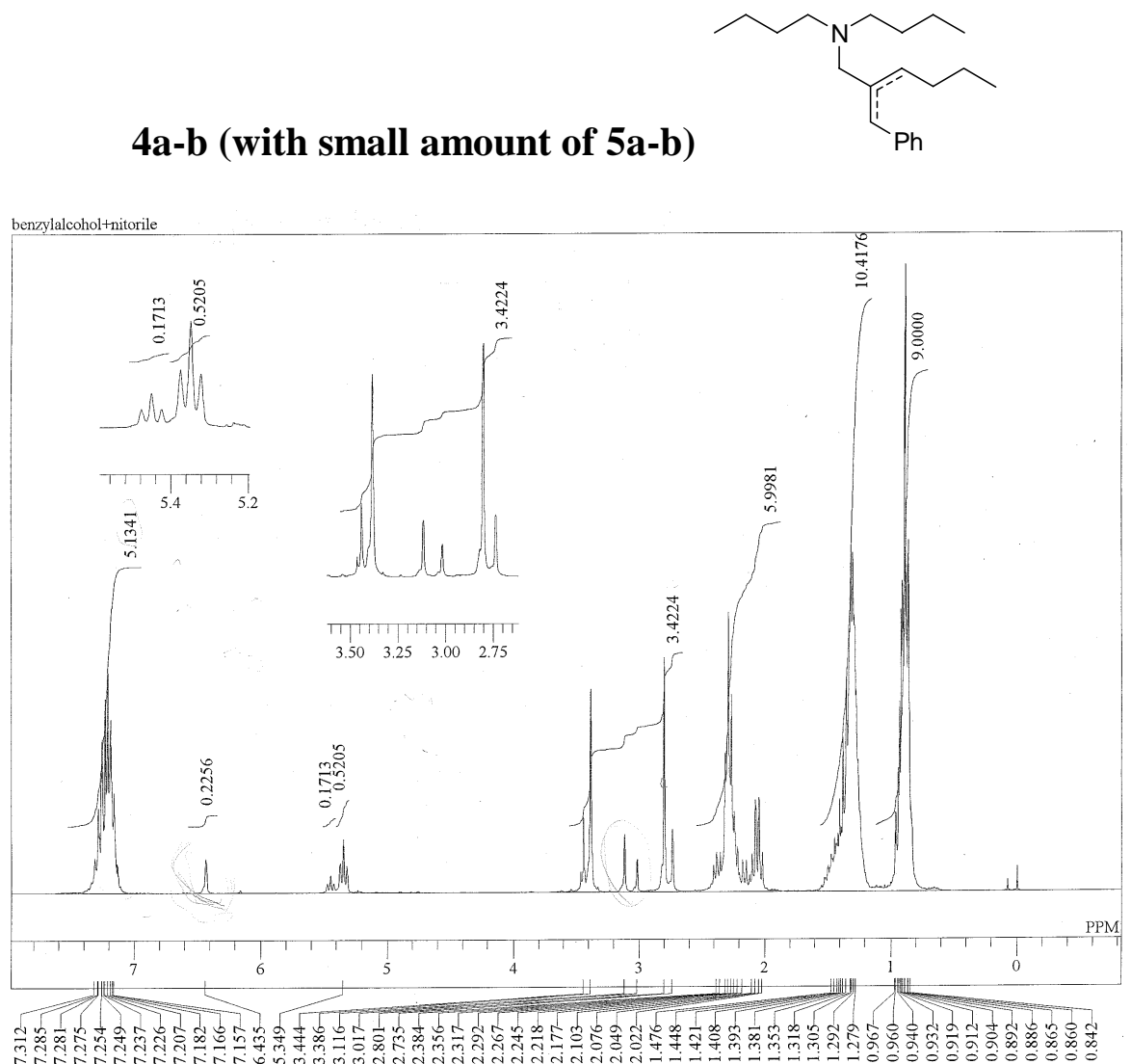


\section{4d (with small amount of 5d)}
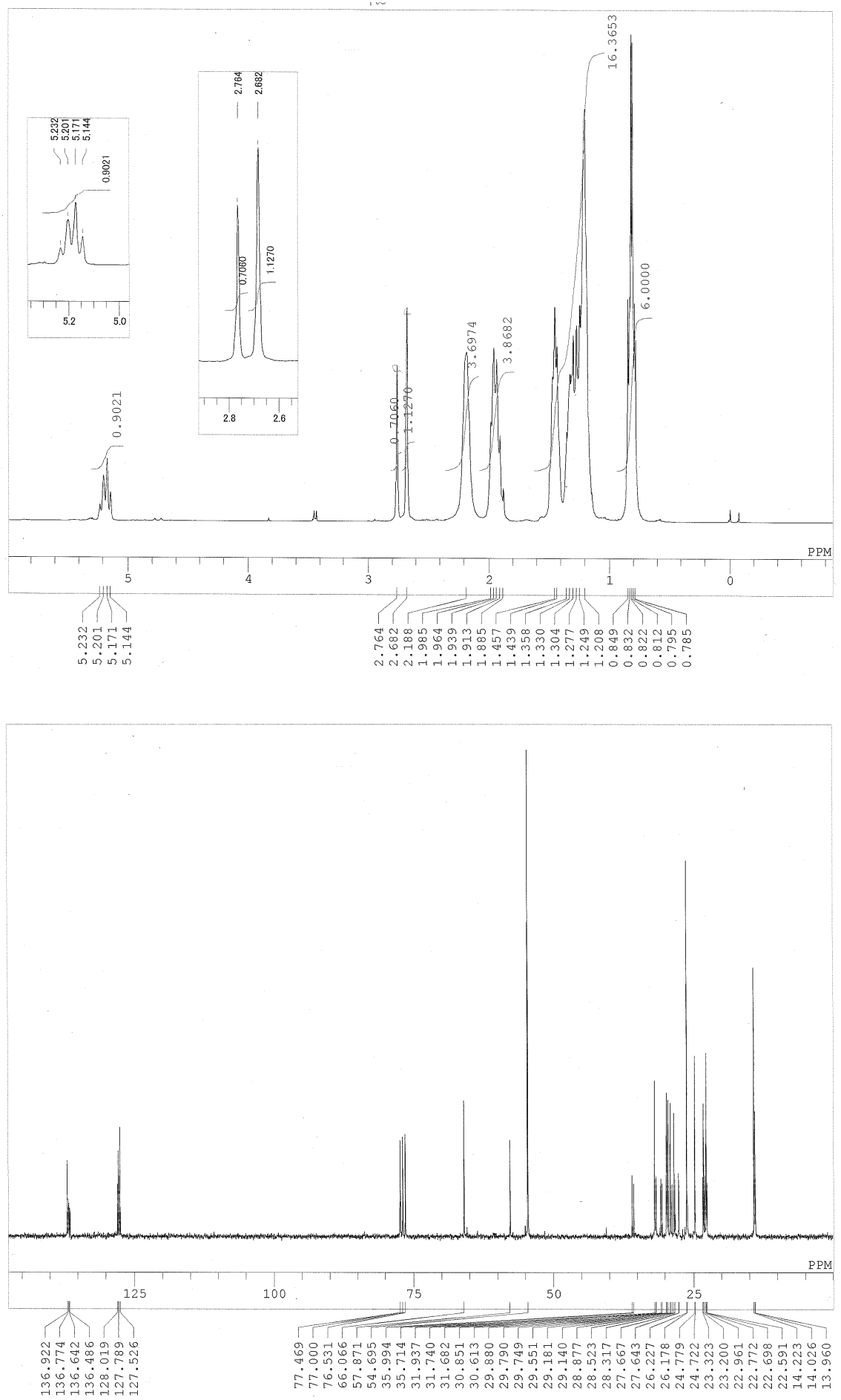


\section{$4 e$ (with small amount of 5e)}

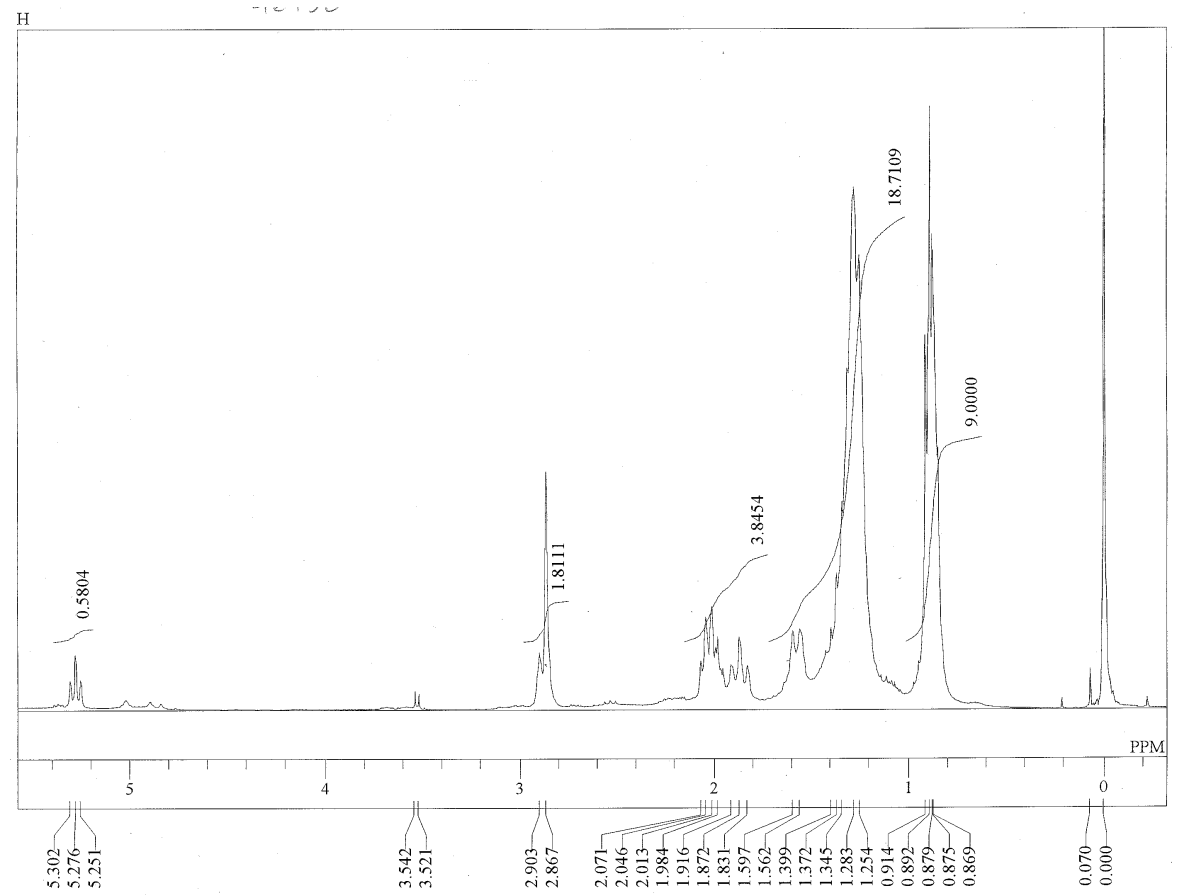


4f (with small amount of $5 f$ )
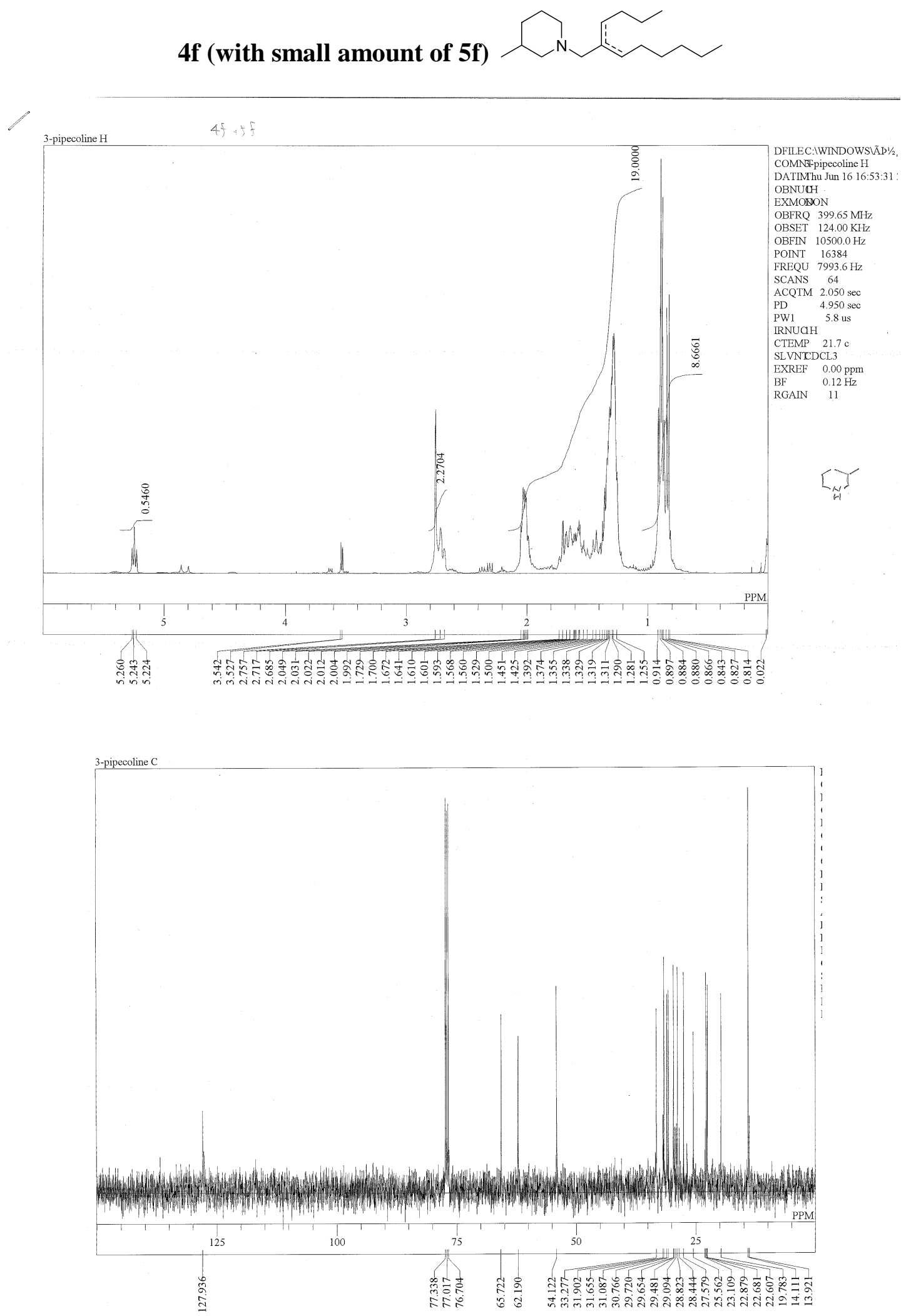
$4 \mathrm{~g}$ (with small amount of $5 \mathrm{~g}$ )
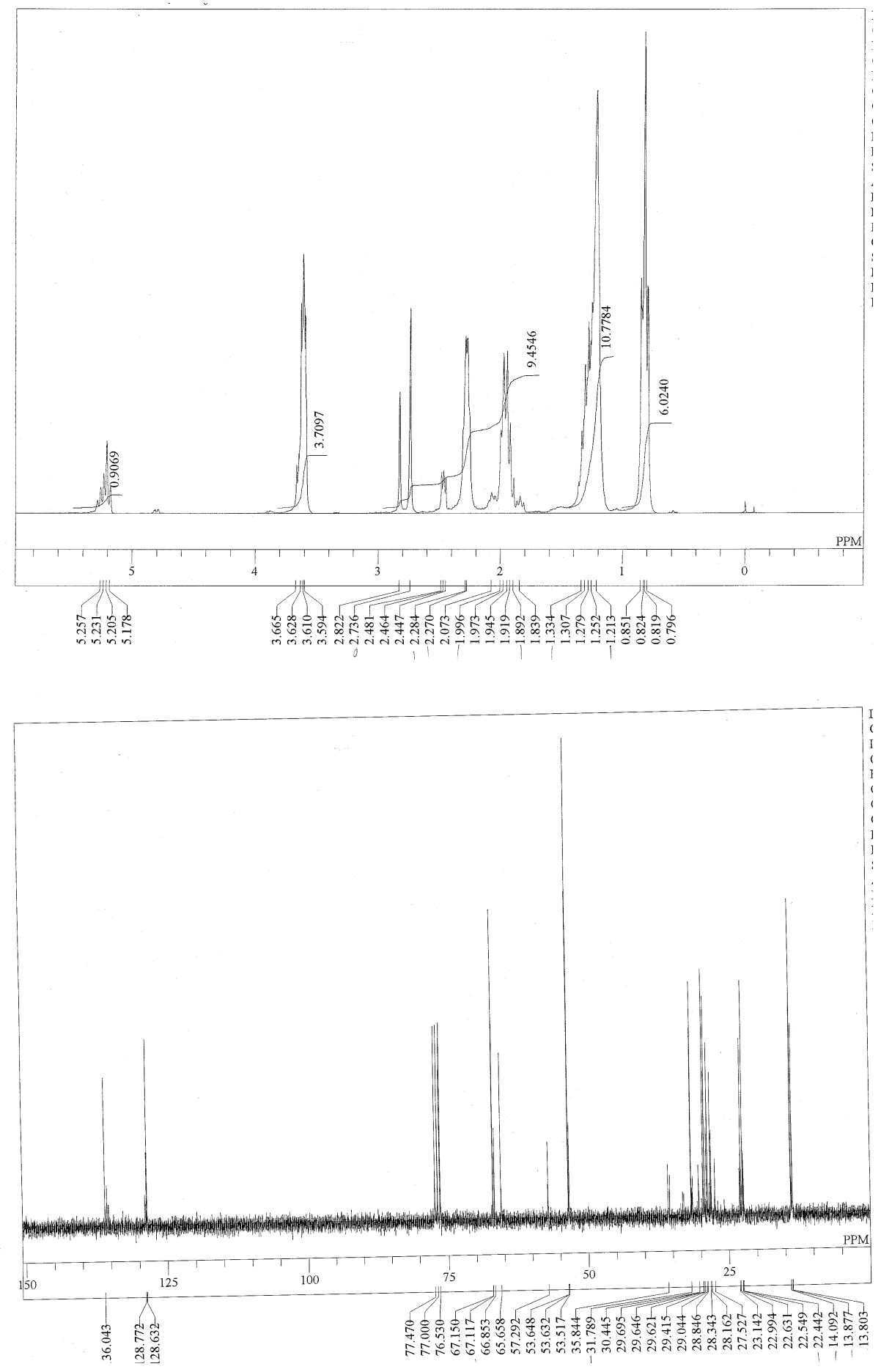
4h (with small amount of 5h)
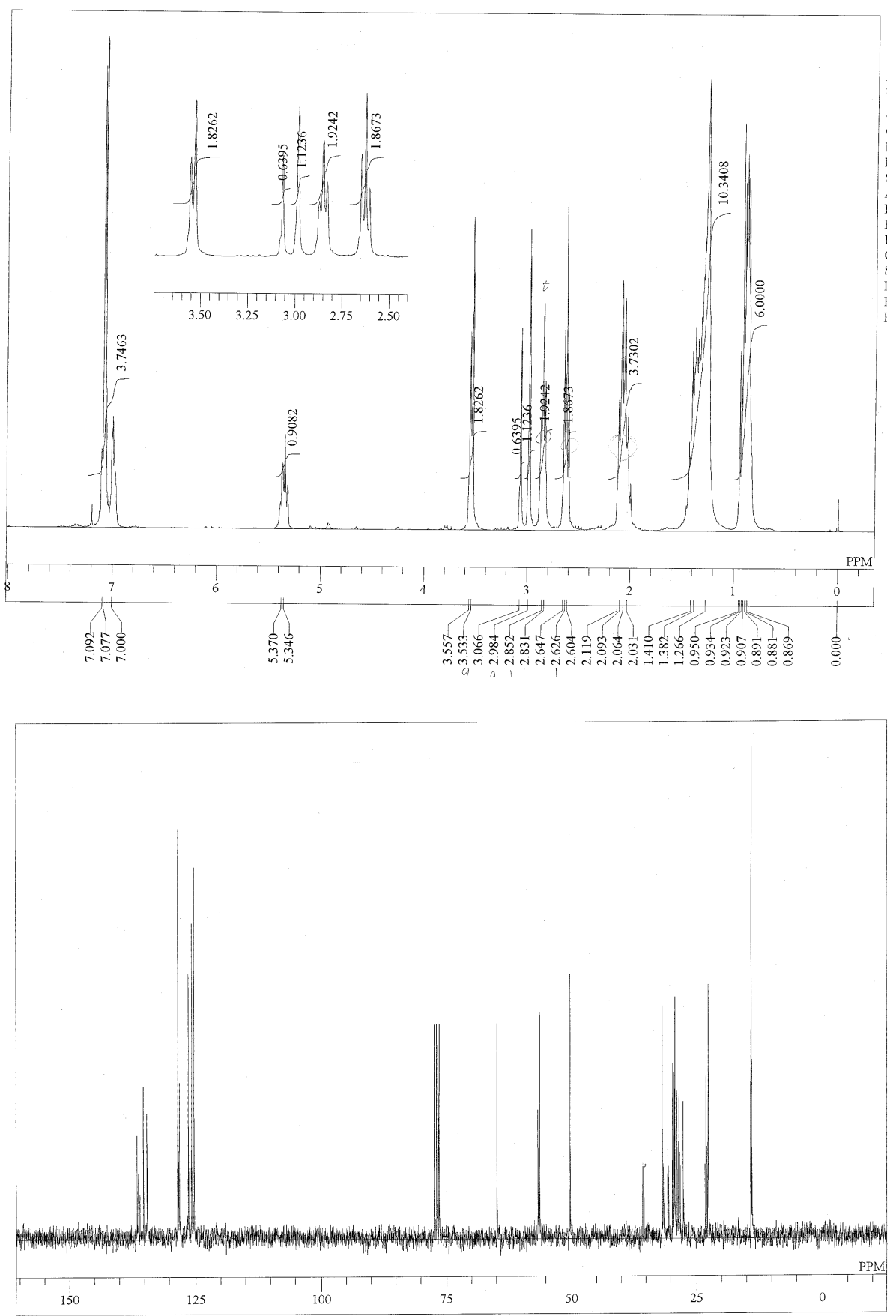